\title{
Predator-prey interactions between droplets driven by non-reciprocal oil exchange
}

\author{
Caleb H. Meredith ${ }^{1,7}$, Pepijn G. Moerman ${ }^{2,3,7}$, Jan Groenewold ${ }^{2,4}$, Yu-Jen Chiu', Willem K. Kegel ${ }^{2}$, \\ Alfons van Blaaderen ${ }^{3}$ and Lauren D. Zarzar (1) 1,5,6凶
}

\begin{abstract}
Chemotactic interactions are ubiquitous in nature and can lead to non-reciprocal and complex emergent behaviour in multibody systems. However, developing synthetic, inanimate embodiments of a chemomechanical framework to generate non-reciprocal interactions of tunable strength and directionality has been challenging. Here we show how chemotactic signalling between microscale oil droplets of different chemistries in micellar surfactant solutions can result in predator-prey-like non-reciprocal chasing interactions. The interactions and dynamic self-organization result from the net directional, micelle-mediated transport of oil between emulsion droplets of differing composition and are powered by the free energy of mixing. We systematically elucidated chemical design rules to tune the interactions between droplets by varying the oil and surfactant chemical structure and concentration. Through the integration of experiment and simulation, we also investigated the active behaviour and dynamic reorganization of multidroplet clusters. Our findings demonstrate how chemically minimal systems can be designed with controllable, non-reciprocal chemotactic interactions to generate emergent self-organization and collective behaviours reminiscent of biological systems.
\end{abstract}

\section{T} he emergent behaviours, dynamics and patterns that evolve within multibody systems, such as swarming insects, crowds of people or bacterial colonies, result from non-equilibrium, distance-dependent coupling between group constituents that is often non-reciprocal, and involves both attractive and repulsive interactions ${ }^{1-3}$. An example two-body non-reciprocal interaction is that of predator and prey, where the predator is attracted to the prey, but the prey is repelled by the predator. In living systems, complex chemomechanical feedback networks govern these non-reciprocal interactions and mediate organisms' abilities to sense, respond and interact with neighbours. However, a similar requisite control over the strength and asymmetry of such non-reciprocal interactions in inanimate systems ${ }^{4-7}$ has not been possible to achieve, probably because chemical gradients not only serve to signal communication between group constituents but also to provide the driving force for motion (for example, chemotaxis) ${ }^{8}$. Here we report an experimental framework by which to chemically programme the specificity, directionality and strength of non-reciprocal predator-prey-like chasing and multibody interactions between microscale oil-in-water droplets. The interactions and dynamic self-organization result from the net directional, micelle-mediated transport of oil between emulsion droplets of differing composition and are powered by the free energy of mixing. The non-reciprocal behaviour occurs in a wide variety of oil and surfactant conditions, and we systematically elucidated chemical design rules to tune the interactions between droplets by varying the oil and surfactant chemical structure and concentration. Experimentally estimated two-body interaction parameters successfully predicted and reproduced multibody behaviour in simulations, which suggests that emulsions can serve as a tractable experimental framework for the study of active materials capable of adaptive, life-like, non-equilibrium dynamics. This robust but facile platform lays the groundwork for the design of soft materials with motility-induced self-organization governed by chemical transport and provides a simple physical model to study emergent, collective phenomena in systems with minimal molecular complexity.

\section{Results}

Two-droplet chasing interactions. Emulsions, which are phase-separated dispersions of fluids stabilized by surfactants, have recently emerged as a rich materials platform in which to study non-equilibrium chemotactic interactions ${ }^{9}$. Emulsions persist in a thermodynamically out-of-equilibrium state and are highly dynamic, with molecules being continually exchanged between droplets and the continuous phase ${ }^{10}$. Droplets can also chemotax, that is, move in response to chemically induced interfacial tension gradients through Marangoni flow ${ }^{11-13}$ or by chemophoresis ${ }^{14}$. For viscous droplets, chemophoretic effects are substantial, but for droplets with viscosities comparable to that of the continuous phase, Marangoni flow is expected to dominate ${ }^{15}$ (Supplementary Information section 'Microscopic mechanism behind droplet motion'). Building on the recent discovery that micelle-mediated oil solubilization gradients generate reciprocal repulsion between droplets of like chemistry ${ }^{16,17}$, we wondered whether asymmetric interactions would occur between droplets of oils with very different micellar solubilization profiles. Thus, we first investigated the interactions between microscale droplets of 1-bromooctane (BOct) and ethoxynonafluorobutane (EFB), two fully miscible, dense oils with very low water solubility and anticipated differences in oil-micelle interactions due to varying degrees of fluorination ${ }^{18}$ (Fig. 1a). In a $0.5 \mathrm{wt} \%$ aqueous Triton X-100 (Triton) non-ionic surfactant, a

'Department of Materials Science and Engineering, The Pennsylvania State University, University Park, PA, USA. ${ }^{2}$ Van't Hoff Laboratory for Physical \& Colloid Chemistry, Debye Institute for Nanomaterials Science, Utrecht University, Utrecht, The Netherlands. ${ }^{3}$ Soft Condensed Matter, Debye Institute for Nanomaterials Science, Utrecht University, Utrecht, the Netherlands. ${ }^{4}$ Guangdong Provincial Key Laboratory of Optical Information Materials and Technology, South China Academy of Advanced Optoelectronics, South China Normal University, Guangzhou, People's Republic of China. ${ }^{5}$ Department of Chemistry, The Pennsylvania State University, University Park, PA, USA. ${ }^{\circ}$ Materials Research Institute, The Pennsylvania State University, University Park, PA, USA. ${ }^{7}$ These authors contributed equally: Caleb H. Meredith, Pepijn G. Moerman. $\varpi_{e}$-mail: Idz4@psu.edu 


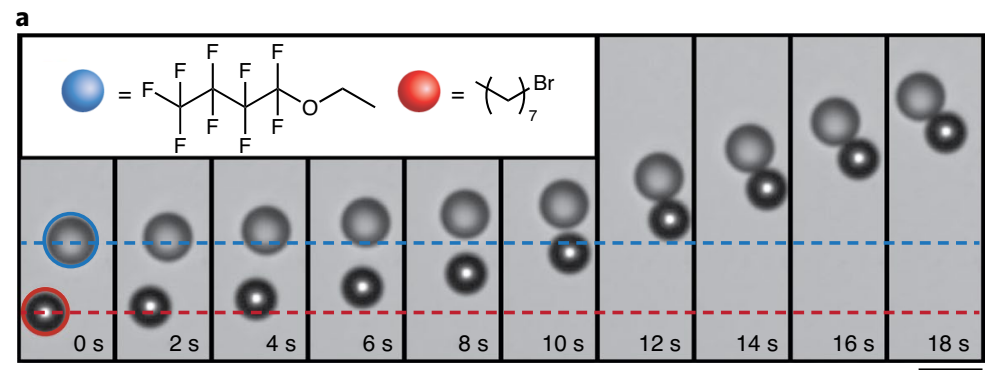

b

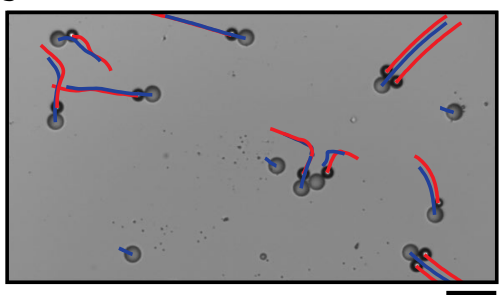

c

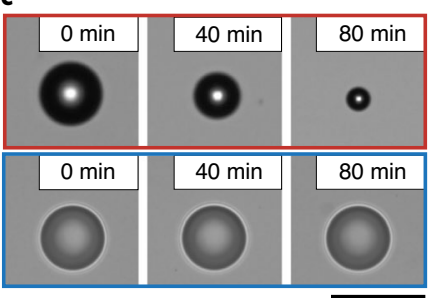

d

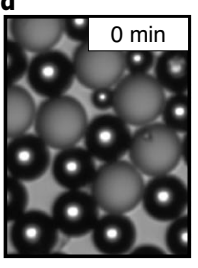

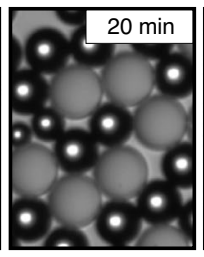

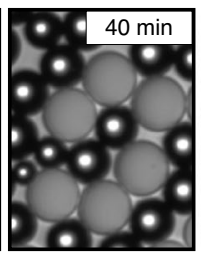

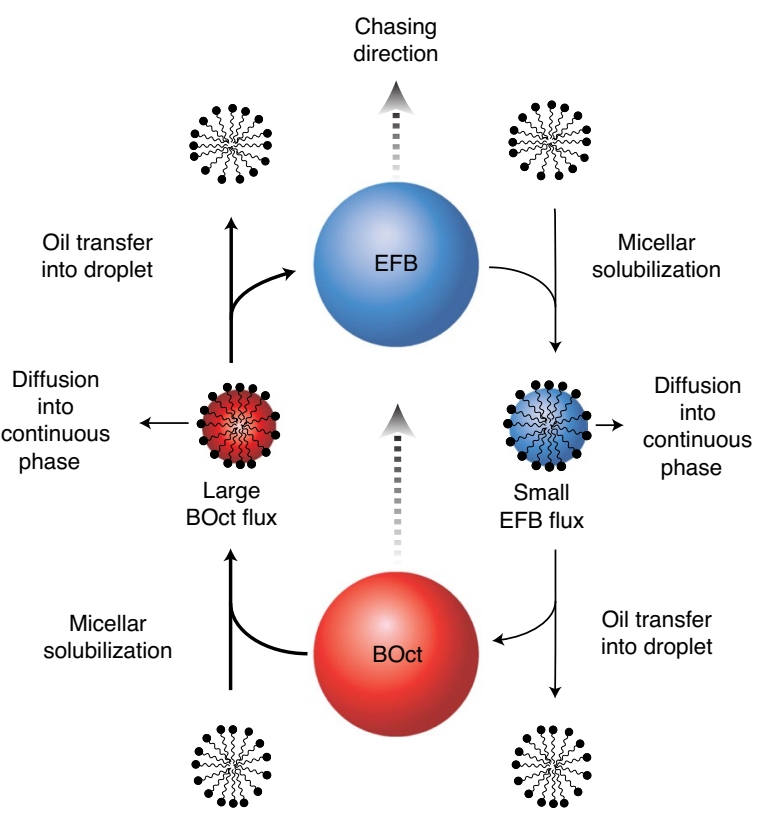

Fig. 1 | BOct droplets chase EFB droplets in aqueous surfactant due to micelle-mediated oil transport. a, Time-sequence frames of a chasing interaction between a BOct predator droplet (outlined in red) and an EFB prey droplet (outlined in blue) in a 0.5 wt\% Triton aqueous solution (Supplementary Video 1). BOct accelerates towards the EFB, and the EFB moves away in response. Eventually, the EFB is 'caught' as the two droplets touch and continue to translate as a pair. Scale bar, $100 \mu \mathrm{m}$. The droplets' initial positions are indicated by the coloured dashed lines. $\mathbf{b}$, Trajectories of BOct drops (red lines) and EFB drops (blue lines) over a period of 30 seconds shows the linear motion of pairs and multidroplet clusters, whereas individual droplets are stagnant. Scale bar, $250 \mu \mathrm{m}$. c, Optical micrographs of an isolated BOct droplet (top) and EFB droplet (bottom) in 0.5 wt\% Triton over time. The diameter of the BOct droplets decreased at a rate of $0.49 \mu \mathrm{m} \mathrm{min}^{-1}$ due to micellar solubilization, but the EFB droplets did not solubilize appreciably. Scale bar, $100 \mu \mathrm{m}$. d, When EFB and BOct droplets were mixed together in 0.5 wt \% Triton, the refractive index of EFB ( $n=1.28)$ increased over time from the uptake of the mobile higher refractive index BOct $(n=1.45)$, and eventually became nearly transparent due to index matching with the aqueous phase ( $n=1.33$ ) and swelling slightly in size. Scale bar, $100 \mu \mathrm{m}$. e, We hypothesize that the non-reciprocal chasing interactions are associated with the asymmetric rates of micelle-mediated oil exchange between droplets, in which the flux of BOct (denoted by bold arrows) is much greater than that of EFB in 0.5 wt\% Triton due to the BOct's higher micellar solubilization rate.

concentration over 25 times Triton's critical micelle concentration, we observed that not only were the droplet interactions asymmetric, but also they were non-reciprocal (Fig. 1a and Supplementary Video 1). Even at initial droplet separation distances of tens of micrometres, BOct 'predator' droplets were attracted to, and accelerated towards, EFB 'prey' droplets, and the EFB prey were repelled by the approaching BOct predators (Fig. 1a). These chases led to the formation of droplet pairs or many-droplet clusters propelled at speeds of upwards of $20 \mu \mathrm{m} \mathrm{s}^{-1}$ with sustained interactions that lasted over an hour (Fig. 1b and Supplementary Video 2). This motion is distinct from the previously reported self-propulsion of individual droplets $^{12,15}$ in that it is a two-body effect that only occurs when a predator and prey droplet meet. No chasing occurred in control experiments in which either the EFB or BOct droplets were replaced with similarly sized polystyrene particles, and isolated droplets of BOct and EFB also did not display notable active behaviour (that is, the self-propulsion speed of individual droplets was, if present, small, approximately $3 \mu \mathrm{m} \mathrm{s}^{-1}$ or less, which is comparable to drift velocities). As BOct droplets solubilized into the continuous phase at a substantially faster rate than did EFB droplets (Fig. 1c), these non-reciprocal chasing interactions appeared to be primarily driven by gradients of BOct. Droplet motion was sustained as long as the BOct predator droplets were not completely solubilized, which usually took over an hour. In the presence of EFB drops, BOct was not only solubilized into the continuous phase, but it was also transferred into the EFB drops, as evidenced by an increase in EFB droplets' refractive index over time (Fig. 1d). Presaturating the surfactant solution with both oils prior to adding both droplets still resulted in chasing interactions. Hence, it appears that the non-reciprocal chasing behaviour is related to asymmetric oil transport between the droplets, in which BOct undergoes micellar solubilization into the continuous phase and is subsequently transferred from the micelles into EFB droplets ${ }^{19,20}$, whereas the transfer of EFB in the reverse direction is minute (Fig. 1e).

Tuning droplet interaction energies. To test this directional transport hypothesis, we aimed to quantify the interaction and displacement energies associated with chasing droplet pairs and examine how these energies vary as a function of parameters that influence micellar transport, such as surfactant concentration and chemical structure. The interaction energy describes whether the interaction is net attractive or repulsive, and the displacement energy quantifies the degree of non-reciprocity of the interaction. At low Reynolds numbers, for any interaction between two droplets with a separation distance $r$, the area under the curve $\delta r / \delta t$ versus $r$ (shaded grey region in Fig. 2a) is proportional to the interaction energy. 


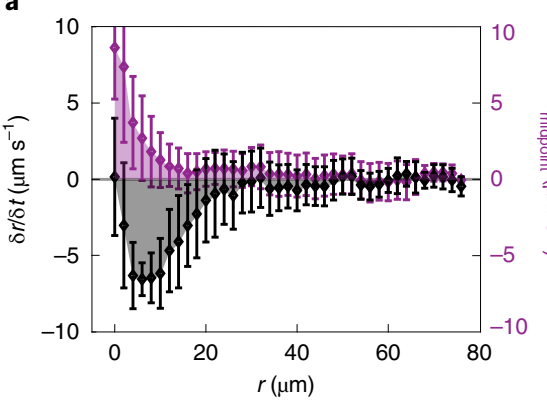

b
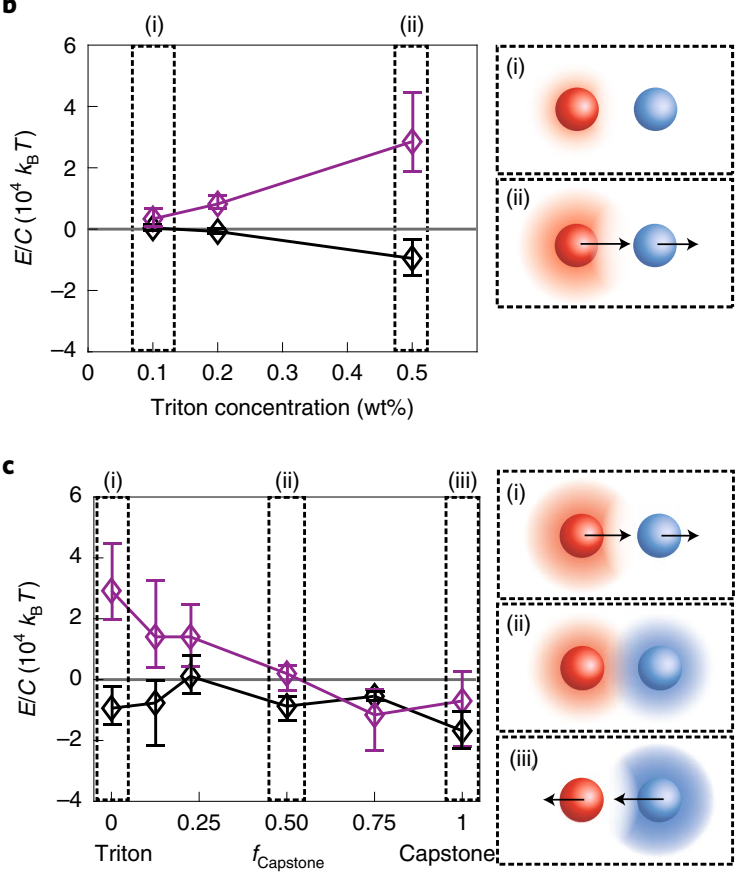

d
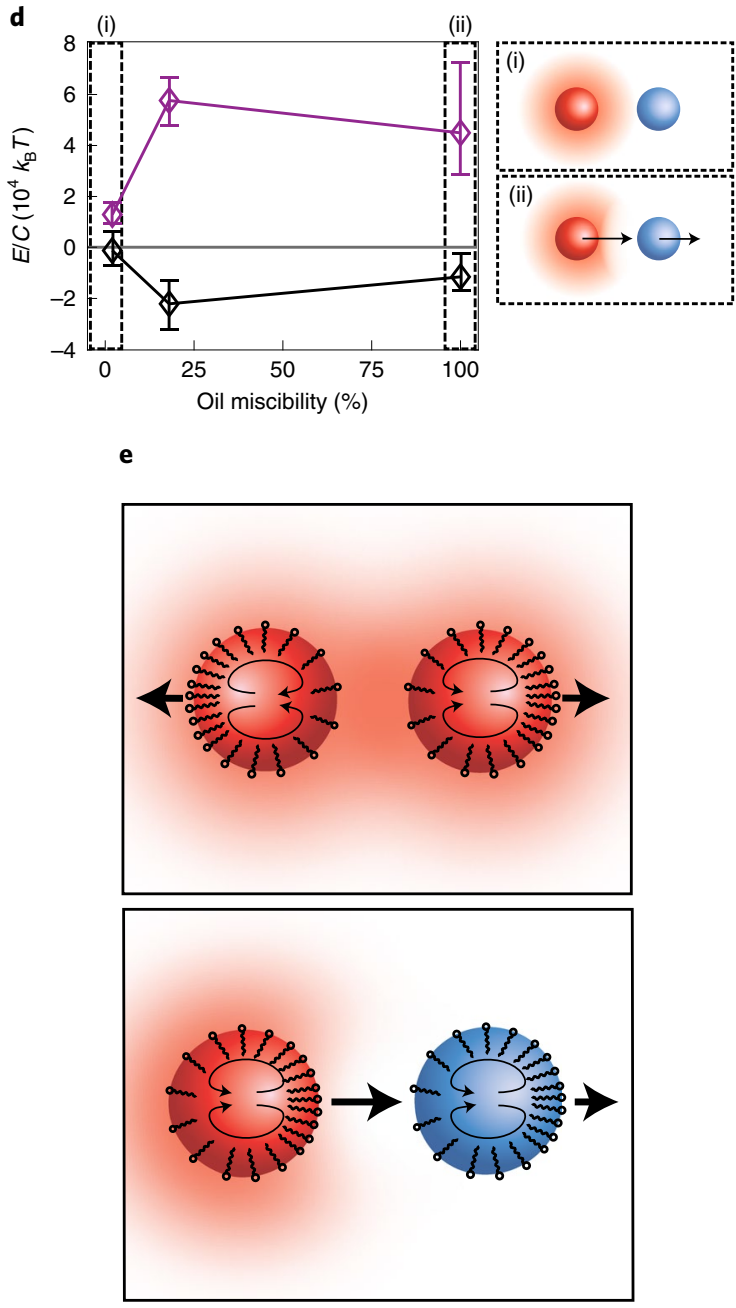

Fig. 2 | Interaction and displacement energies associated with predator-prey droplets are tunable by varying the chemical structure and concentration of surfactant and oil miscibility. $\mathbf{a}$, Interaction and displacement energies (calculated from measurements $r$ and $V_{\text {midpoint }}$ ) for interactions between $B O c t$ (red drop) and EFB (blue drop) in $0.5 \mathrm{wt} \%$ Triton; each data point represents the average and standard deviation from at least three independent droplet encounters. The interaction and displacement energies are proportional to the grey and purple shaded regions, respectively. As defined, a positive displacement energy indicates that BOct chases EFB, whereas a negative displacement energy corresponds to the reverse chase direction. $\mathbf{b}$, The interaction energy (black line) and displacement energy (purple line) were measured under varying concentrations of Triton by creating plots like that shown in a for each droplet encounter; the shaded grey and shaded purple areas were multiplied by a drag constant, which we take to be the Stokes' drag multiplied by a dimensionless factor $(C)$ to account for contributions to the drag constant from droplet internal flows and substrate proximity effects, to yield energies, $E$, on the order of $10^{4} k_{B} T$, where $T=298 \mathrm{~K}$. Each data point represents an average from three droplet encounters and the error bars represent the highest and lowest measurements. The schematics (right) illustrate the solubilizate gradients and the chasing directions for regions ( $i$ ) and (ii). As the Triton concentration increased, the solubilization rate of BOct increased from 0.09 to $0.49 \mu \mathrm{m} \mathrm{min}^{-1}$, and the interactions were stronger. c, Interaction and displacement energies for BOct and EFB as a function of Capstone $\left(f_{\text {Capstone }}\right)$, which corresponds to the volume fraction of 3 wt $\%$ Capstone in a mixture with $0.5 \mathrm{wt} \%$ Triton. The schematics (right) represent the relative oil gradients around each drop and chasing directions for regions (i)-(iii). Each data point represents an average from three droplet encounters and the error bars represent the highest and lowest measurements. d, Displacement and interaction energies as a function of the prey fluorinated oil miscibility with predator BOct in 0.5 wt $\%$ Triton. The fluorinated oils were perfluorohexane (region (i)), methoxyperfluorobutane (middle datapoints, illustration not shown) and EFB (region (ii)). Refer to Methods section, 'Measurement of refractive indices and oil miscibility'. The schematics (right) illustrate the oil gradients and chasing directions. Each data point represents an average from three droplet encounters with error bars representing the highest and lowest measurements. e, Schematics of how micelle-mediated oil transport couples with Marangoni flow. Top and bottom: predator droplets (red) produce oil-filled micelles and repel all droplet neighbours because the oil solubilizate decreases the surfactant's ability to stabilize oil-water interfaces. Bottom: prey droplets (blue) uptake the predator's solubilized oil from micelles and so attenuate the oil gradients and attract predators. The prey is still repelled by the predator, which leads to chasing.

A negative interaction energy indicates a net attraction (for example, a successful chase in which the predator catches the prey), whereas a positive interaction energy indicates repulsion (for example, a failed chase, in which the predator does not catch the prey). As the droplet interactions are non-reciprocal, not only does $r$ change over time, but the midpoint between the droplets also moves with velocity
$V_{\text {midpoint }}$ as the predator approaches the prey. We used the area under the $V_{\text {midpoint }}$ versus $r$ curve (shaded purple region in Fig. 2a), which is proportional to the displacement energy, to quantify the degree of non-reciprocity. As defined in Fig. 2a, a positive displacement energy indicates that BOct chases EFB, whereas a negative displacement energy corresponds to the reverse chasing direction. By multiplying 
a

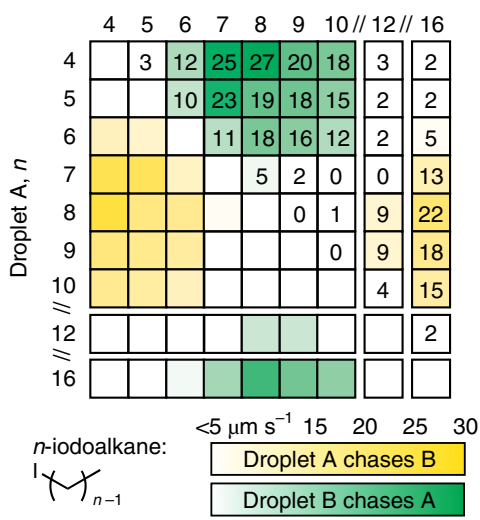

b

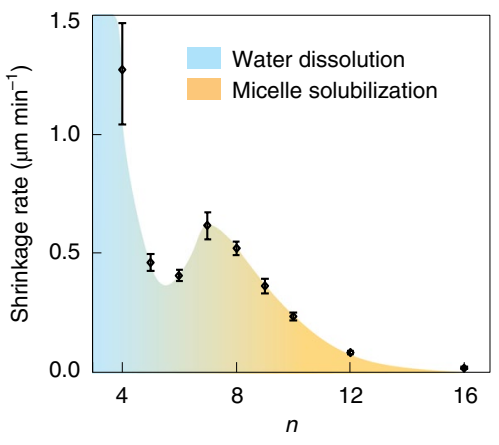

Droplet B, $n$

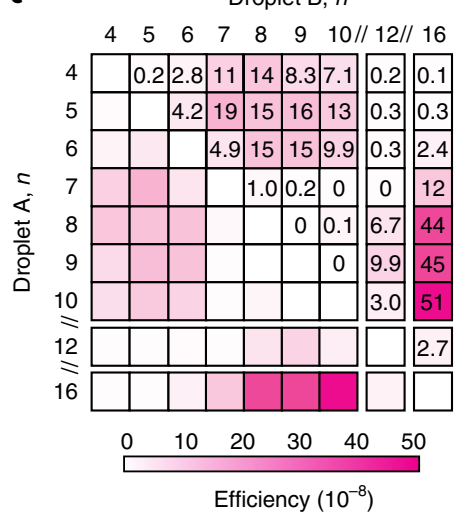

Fig. 3 | Oil molecular structure influences the direction, speed and efficiency of chasing between droplets. a, Tabulated average chasing speeds in 0.5 wt\% Triton for all pairwise combinations of 1-iodo- $n$-alkane droplets, identified by carbon number $n=4-16$. Chase speed is defined as the speed of the predator-prey pair once contact between droplets was made, and the speed reported is the average of three chases. Colour coding in the yellow and green regions indicates the chasing direction between droplet $A$ and droplet $B$ of defined carbon number $n$. Pairs for which interactions were weak (with speeds below $5 \mu \mathrm{m} \mathrm{s}^{-1}$ ) or reciprocal (left-to-right centre diagonal) were left white. $\mathbf{b}$, Solubilization rates (change in drop diameter over time) of the iodo- $n$-alkanes were measured for isolated droplets in $0.5 \mathrm{wt} \%$ Triton. For each value of $n$, the solubilization rate of three independent droplets was measured and the average, maximum and minimum are reported in the plot. Two solubilization pathways exist: direct water dissolution (cyan region, more likely for oils with

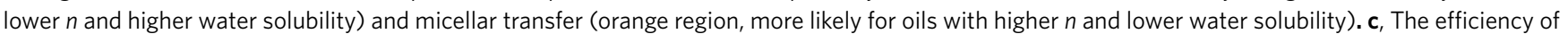
droplet chasing for each pair of iodo- $n$-alkanes was calculated based on the combined rates of oil loss for predator and prey (that is, the chemical energy input) and the resulting speed of the chasing droplet pair (that is, the mechanical energy output). Highest efficiencies (dark pink areas) were found for oil pairs in which the predators primarily solubilized by the micellar transfer pathway and the prey did not solubilize appreciably so as to minimize oil lost to the water and maximize the asymmetry of the predator-prey interaction.

the areas under these two curves in Fig. 2a by the Stokes' drag and introducing a dimensionless constant $C$ to account for contributions to the drag constant from droplet internal flows and substrate proximity effects, we calculated the interaction and displacement energies for any two-body droplet interaction. For chasing BOct and EFB droplets in $0.5 \mathrm{wt} \%$ Triton, these energies were on the order of $10^{-18} \mathrm{~J}$, or $10^{4} k_{\mathrm{B}} T$, where $k_{\mathrm{B}}$ is the Boltzmann constant and $T$ the temperature in Kelvin (taken as $298 \mathrm{~K}$ ) (Fig. 2b-d). Please refer to the Supplementary Information section 'Quantification of interaction energy and displacement energy for two-body droplet chasing' for further details of these calculations.

Having established a method by which to quantitatively compare the interaction and displacement energies associated with any two-body chasing interaction, we examined how those energies change with surfactant concentration, surfactant chemical structure and oil chemical structure. At each set of experimental conditions, we analysed multiple (at least three) independent chasing interactions. As the concentrations of Triton were increased from $0.1 \mathrm{wt} \%$ to $0.5 \mathrm{wt} \%$ (which are all concentrations above the critical micelle concentration), the magnitude of both the interaction and displacement energies associated with EFB and BOct encounters increased, which indicates that a higher concentration of micelles is associated with a greater flux of BOct and stronger chasing interactions (Fig. 2b). Replacing Triton with 3.0 wt\% Capstone FS-30 (Capstone), a non-ionic fluorosurfactant that preferentially solubilizes the fluorinated $\mathrm{EFB}$, reversed the chasing direction such that EFB was the predator and BOct the prey (Fig. 2c and Supplementary Video 3). This reversal in chasing direction is indicated by the sign reversal of the displacement energy (Fig. 2c) and shows that the net direction of micelle-mediated oil transport determines the chasing direction. The allowed exchange of EFB to BOct in Capstone is supported by the observed index matching of a BOct droplet over time (Extended Data Fig. 1). Mixing $0.5 \mathrm{wt} \%$ Triton and $3.0 \mathrm{wt} \%$ Capstone in varying ratios allowed a continuous tuning of the chasing speed and direction of BOct and EFB pairs (Fig. 2c). If both oils solubilized at appreciable rates in a surfactant mixture (for example,
Figure $2 \mathrm{c}(\mathrm{ii})$ ), then no chasing occurred, which is consistent with the hypothesis that a net directional oil transport between droplets is required. When EFB was replaced with methoxyperfluorobutane, another fluorinated oil with a partial miscibility with BOct, similar chasing interactions in $0.5 \mathrm{wt} \%$ Triton occurred; however, when EFB was replaced with perfluorohexane, a fluorinated oil with very little miscibility with BOct, there was no chasing (Fig. 2d). As none of the fluorinated oils solubilized appreciably in $0.5 \mathrm{wt} \%$ Triton, this influence of oil miscibility on chasing suggests that favourable oil mixing is also essential for the predator-prey interaction to occur.

Mechanism for chasing interactions. Our observations regarding the influence of micelle-mediated oil transport on the chasing interactions suggest the following underlying Marangoni-flow-driven mechanism for non-reciprocal droplet behaviour (Fig. 2e): (1) Predator droplets produce oil-filled micelles that repel all droplet neighbours because the oil solubilizate decreases the surfactant's ability to stabilize oil-water interfaces, raising the interfacial tension (Extended Data Fig. 2). Gradients of oil-filled micelles thus increase the interfacial tension on the sides of droplets that face nearby predators, which causes an asymmetric surfactant distribution on the droplets' surface, which drives motion away from the predator's signal via Marangoni flow. Thus, the two predator droplets repel each other symmetrically, and this motion leads to an overall decrease in the droplets' interfacial energy (Fig. 2e, top). (2) Prey droplets uptake the predator's solubilized oil from micelles, which 'frees' surfactant molecules that can more efficiently stabilize oil-water interfaces. The oil uptake by the prey thus attenuates the solubilizate gradients, which decreases the interfacial tension of the predator droplet on the side closer to the prey; this causes the predator droplet to move towards the prey (Fig. 2e, bottom). The prey droplet still attempts to flee the predator, driven towards regions of solution that contain lower concentrations of solubilized oil, as a means to reduce interfacial energy. This experimental description is analogous to the 'source' and 'sink' framework often used to achieve predator-prey interactions in theoretical models ${ }^{7,21}$; here, the BOct 
a

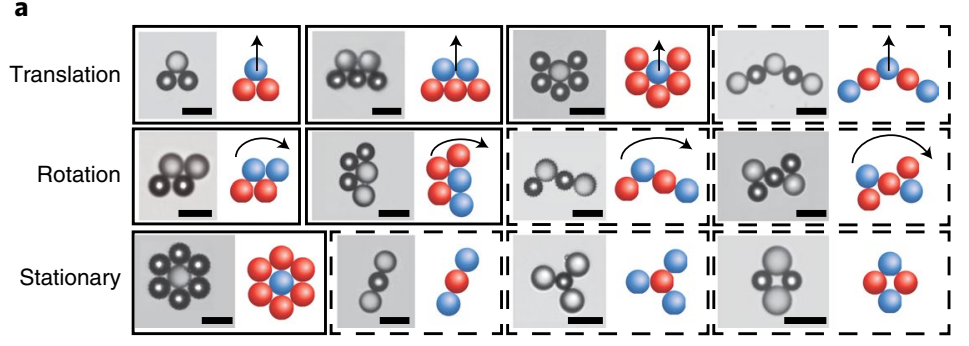

b

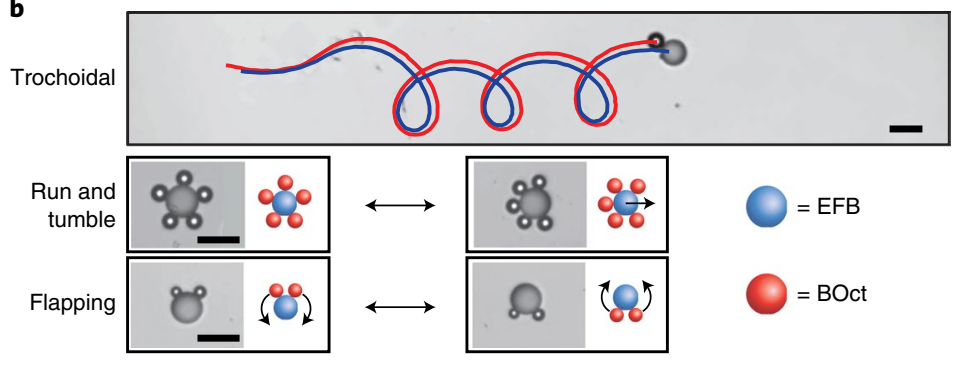

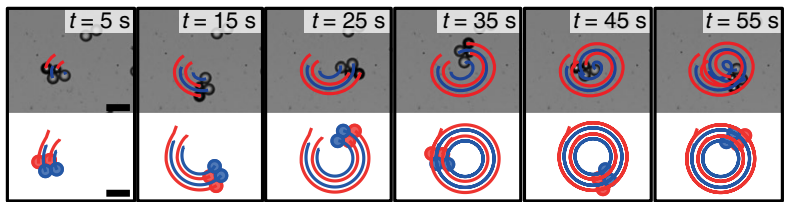

d
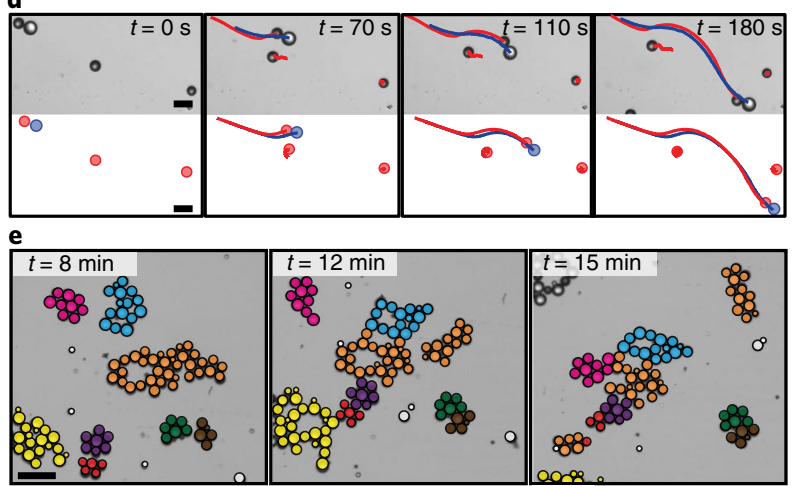

Fig. 4 | Multibody non-reciprocal interactions between droplets cause emergent assembly and disassembly dynamics that are predictable based on measured two-body interactions. a, In mixtures that contain a high number density of BOct and EFB droplets in 0.5 wt $\%$ Triton, multidroplet clusters assembled via non-reciprocal interactions and exhibited movements (indicated by arrows) dependent on the cluster geometry. A short-ranged sticking attraction between droplets led to the formation of cluster geometries that would be otherwise unstable to formation (bordered by dashed lines). Scale bar, $100 \mu \mathrm{m}$. b. As the diameter of BOct decreased below approximately $40 \mu \mathrm{m}$, the droplets became self-propelled and started to migrate along the surface of the prey, which led to the formation of trochoidal trajectories, run-and-tumble dynamics and flapping motions. Scale bar, $100 \mu \mathrm{m}$ (Supplementary Information section 'Trochoidal trajectories' and Supplementary Video 7). c, Simulations reproduce the observed cluster dynamics of EFB (blue) and BOct (red) using the initial droplet positions and interaction parameters obtained from their two-body interaction potential measurements. Shown is the rotation of a four-droplet cluster that contains two predators and two prey (Supplementary Video 8). Scale, 100 $\mu$ m. $\mathbf{d}$, Solute-mediated interactions operate at distances of upwards of $50 \mu \mathrm{m}$, which causes individual droplets (here, BOct) to act as signalling posts that direct the movement of a nearby chasing pair of BOct and methoxyperfluorobutane, as shown in experiment and simulation (Supplementary Video 9 ). Scale bar, 100 $\mu$ m. e, Droplets of BOct and EFB in 0.5 wt\% Triton continuously assemble and reorganize over time (Supplementary Video 6). Droplets in the same cluster at $t=8 \mathrm{~min}$ are labelled in the same colour, isolated droplets not in a cluster at $t=8 \mathrm{~min}$ are labelled in white and droplets not in the frame at $t=8 \mathrm{~min}$, but that entered the field of view at later time points were unlabelled. As evidenced by the colour mixing, these clusters were continuously rearranged and reformed from collisions and solution-mediated interactions. Scale bar, $200 \mu \mathrm{m}$.

drop is the source (for example, an emitter of the chemical signal) and the EFB drop is the sink (for example, a consumer of the chemical signal). The source and sink act together to modify the chemical gradients of solubilizate that modulate interfacial tension gradients, which creates a mechanism for chemomechanical feedback and leads to non-reciprocal behaviour. Note that the terms predator and prey may suggest that the predator droplet consumes the prey droplet, but this is not the case. The predator chases the prey, but there is net oil transport from the predator to the prey droplet.

To test the generality of this proposed mechanism among oils of a more similar chemistry, we examined the chasing direction and speed of pairs of 1 -iodo- $n$-alkane microdroplets (Fig. 3a). Iodo- $n$-alkanes (hereafter identified by the carbon number, $n=4-16)$ were chosen for these systematic studies instead of $n$-alkanes or 1-bromo- $n$-alkanes for ease of experimentation because they are denser than the aqueous continuous phase and remain liquid at room temperature for all chain lengths up through to 1-iodohexadecane. An oil-soluble dye was used to differentiate between the iodoalkane droplets in the chasing pairs (which are otherwise indistinguishable optically due to their similar refractive index) as well as confirm that transfer of droplet contents still occurs (Extended Data Figs. 3 and 4). Chasing between droplets of many oil combinations was observed (Fig. 3a) and, interestingly, even a structural difference as minute as a single methylene bridge $\left(-\mathrm{CH}_{2}-\right)$ could be sufficient to drive chasing (for example, 1-iodoheptane chases 1-iodohexane; Supplementary Video 4). By dispersing tracer particles in each of the oils and the surfactant solution, we directly visualized the flow patterns formed inside and outside both predator and prey droplets as they chased (Supplementary Video 5 and Extended Data Fig. 5). As highlighted by the green and yellow regions in Fig. 3a, the designation of predator and prey for each pairing was not simply a function of relative oil chain length; oil droplets of both the shortest $(n=4-6)$ and longest ( $n=12$ and 16) chain lengths tended to be prey, whereas intermediate chain lengths $(n=7-10)$ tended to be predators (Fig. 3a). Hydrocarbon oils exhibited qualitatively similar chasing trends as a function of chain length (for example, octane chased both hexane and hexadecane in $0.5 \mathrm{wt} \%$ Triton; Extended Data Fig. 3c). Oil solubilization rates for isolated droplets (Fig. 3b) also did not decrease monotonically with increasing $n$, as would be expected in the absence of surfactant ${ }^{22}$, which suggests that oil solubilization kinetics, and hence the chasing direction, are influenced by two transport pathways ${ }^{10,23,24}$ : (1) direct water dissolution (dominant for oils with lower $n$ and higher water solubility) and (2) direct micellar transfer (dominant for oils with higher $n$ and lower water solubility). Droplets of oils for which transfer is dominated by pathway (1) were always prey (for example, $n=4$ and 5) even if they solubilized faster than their predators. If the solubilization of both oils in a pair was dominated by the micellar transfer of pathway (2) (that is, $n=7$ and higher), then the relative rates of oil solubilization did qualitatively correlate with chasing direction and the predator was always solubilized more rapidly than the prey. If neither oil in a pair solubilized appreciably by pathway (2), then the interactions were weak to non-existent. These observations lead to an important 
insight: the direction and speed of chasing is influenced not only by the direction and rate of net oil transport, but also by the specific molecular pathway for that transport, where pathway (1) does not contribute as substantially to the Marangoni effect driving chasing as does pathway (2). Accordingly, the highest chasing efficiencies (Fig. 3c; see Supplementary Information section 'Efficiency of chasing driven by oil exchange' for a description of the calculation) were found for oil pairs in which the predator droplets were preferentially solubilized by the direct micellar transport pathway (2) (for example, $n=7$ or higher) and where the prey did not solubilize appreciably $(n=16)$ so as to maximize the asymmetry of the interaction and minimize the total oil lost to the continuous phase. Maximum efficiencies obtained for the iodoalkane droplet pairs were on the order of $10^{-7}$, which is approximately two orders of magnitude higher than the efficiency of the well-studied, self-electrophoretic bimetallic motors ${ }^{25}$.

Multidroplet interactions and collective behaviours. In experiments with a high number density of droplets, the formation of chasing pairs was the first step in a cascade of collisions and reorganizations, which resulted in the assembly of myriad clusters with diverse structures and dynamics (Fig. 4a and Supplementary Video 6). Multibody interactions between BOct and EFB oil droplets in $0.5 \mathrm{wt} \%$ Triton generated clusters that rotated, translated or remained stationary depending on their geometry (Fig. 4a). A short-ranged sticking attraction between droplets ${ }^{26,27}$ led to the formation of clusters that would otherwise have been unstable due to multiple prey droplets fleeing in different directions from the same predator (for example, the clusters bordered by the dashed boxes in Fig. 4a). Although varying the droplet sizes did not qualitatively change the two-body chasing direction, droplet size did affect the multibody behaviour. Not only did the relative diameter of predator and prey impact the packing geometry of the clusters, but BOct droplets with diameters below approximately a $40 \mu \mathrm{m}$ diameter became self-propelled ${ }^{28}$ (Extended Data Fig. 6) and continued to translate around the EFB drop to which they were attached. Such predator self-propulsion resulted in trochoidal trajectories (for two-body interactions; Supplementary Information section 'Trochoidal trajectories' and Extended Data Fig. 7), and in flapping and run-and-tumble dynamics (for multibody clusters) (Fig. 4b and Supplementary Video 7). To investigate the extent to which the multibody cluster dynamics can be understood by summation of the measured two-body droplet interactions, we compared our experiments with simulations (see Supplementary Information section 'Quantification of interaction parameters for simulations' for details). The circular and linear motions of clusters could be readily reproduced in simulations by using the initial positions of the droplets and two-body interaction parameters estimated from experiments as inputs (Fig. 4c,d and Supplementary Videos 8-10). Owing to long-range solute-mediated interactions, neighbouring clusters interact and influence each other's structure and trajectory. For example, both experiment and simulation show that individual droplets can act as chemical signalling posts to effectuate turns and reorientations of nearby chasing pairs (Fig. 4d, and Supplementary Video 9) or cause neighbouring clusters to disassemble (Supplementary Video 10). Thus, in contrast to static, self-assembled equilibrium structures, these droplet clusters were dynamically disassembled, rearranged and reformed over time through non-equilibrium self-organization mediated by solute gradients and physical collisions ${ }^{29}$ (Fig. 4 e and Supplementary Video 6).

\section{Discussion}

We have established a facile, robust platform to produce controllable, non-reciprocal predator-prey interactions between oil-in-water droplets powered by the free energy of mixing and achieved by combining micellar oil exchange and Marangoni flow.
Only four chemical components are necessary to tune the predator-prey interactions in this system: two chemically distinct, but miscible oils, a surfactant that preferentially solubilizes one of the oils in micelles, and water. The chasing speed, direction and efficiency are governed by the rate and direction of micelle-mediated oil exchange between droplets, which can be rationally controlled by varying the oil chemical structure, surfactant chemical structure or surfactant concentration. Many-body interactions were accurately described by simulations based on measured two-body droplet interactions, which suggests that this experimental platform may be suitable to test theoretically predicted aspects of dynamic collective behaviour. Further routes to control droplet chasing through the tailoring of solubilizate-micelle intermolecular interactions $^{30}$ may enable selective chemical transport across multiple orthogonal communication pathways for more complex networks of interacting droplets ${ }^{31}$. We expect that this experimental platform and the concomitant chemical design rules laid forth will pave the way to developing inanimate systems with dynamic or life-like adaptive organizations and functionalities ${ }^{32-35}$ that are not accessible through equilibrium assembly.

\section{Online content}

Any methods, additional references, Nature Research reporting summaries, source data, extended data, supplementary information, acknowledgements, peer review information; details of author contributions and competing interests; and statements of data and code availability are available at https://doi.org/10.1038/ s41557-020-00575-0.

Received: 26 December 2019; Accepted: 12 October 2020;

Published online: 16 November 2020

\section{References}

1. Vicsek, T. \& Zafeiris, A. Collective motion. Phys. Rep. 517, 71-140 (2012).

2. Czirók, A., Ben-Jacob, E., Cohen, I. \& Vicsek, T. Formation of complex bacterial colonies via self-generated vortices. Phys. Rev. E 54, 1791 (1996).

3. Bellomo, N. \& Soler, J. On the mathematical theory of the dynamics of swarms viewed as complex systems. Math. Models Methods Appl. Sci. 22, 1140006 (2012).

4. Cira, N. J., Benusiglio, A. \& Prakash, M. Vapour-mediated sensing and motility in two-component droplets. Nature 519, 446-450 (2015).

5. Niu, R., Palberg, T. \& Speck, T. Self-assembly of colloidal molecules due to self-generated flow. Phys. Rev. Lett. 119, 028001 (2017).

6. Theurkauff, I., Cottin-Bizonne, C., Palacci, J., Ybert, C. \& Bocquet, L. Dynamic clustering in active colloidal suspensions with chemical signaling. Phys. Rev. Lett. 108, 268303 (2012).

7. Usta, O. B., Alexeev, A., Zhu, G. \& Balazs, A. C. Modeling microcapsules that communicate through nanoparticles to undergo self-propelled motion. ACS Nano 2, 471-476 (2008).

8. Sengupta, A., Kruppa, T. \& Löwen, H. Chemotactic predator-prey dynamics. Phys. Rev. E 83, 031914 (2011).

9. Lach, S., Yoon, S. M. \& Grzybowski, B. A. Tactic, reactive, and functional droplets outside of equilibrium. Chem. Soc. Rev. 45, 4766-4796 (2016).

10. Christian, S. D. \& Scamehorn, J. F. Solubilization in Surfactant Aggregates (CRC Press, 1995).

11. Schmitt, M. \& Stark, H. Swimming active droplet: a theoretical analysis. Europhys. Lett. 101, 44008 (2013).

12. Maass, C. C., Krüger, C., Herminghaus, S. \& Bahr, C. Swimming droplets. Annu. Rev. Condens. Matter Phys. 7, 171-193 (2016).

13. Lagzi, I., Soh, S., Wesson, P. J., Browne, K. P. \& Grzybowski, B. A. Maze solving by chemotactic droplets. J. Am. Chem. Soc. 132, 1198-1199 (2010).

14. Anderson, J. L. Colloid transport by interfacial forces. Annu. Rev. Fluid Mech. 21, 61-99 (1989).

15. Izri, Z., van der Linden, M. N., Michelin, S. \& Dauchot, O. Self-propulsion of pure water droplets by spontaneous Marangoni-stress-driven motion. Phys. Rev. Lett. 113, 248302 (2014).

16. Moerman, P. G. et al. Solute-mediated interactions between active droplets. Phys. Rev. E 96, 032607 (2017).

17. Jin, C., Krüger, C. \& Maass, C. C. Chemotaxis and autochemotaxis of self-propelling droplet swimmers. Proc. Natl Acad. Sci. USA 114, 5089-5094 (2017).

18. Gladysz, J. A., Curran, D. P. \& Horváth, I. T. Handbook of Fluorous Chemistry (John Wiley \& Sons, 2006). 
19. De Smet, Y., Deriemaeker, L. \& Finsy, R. Ostwald ripening of alkane emulsions in the presence of surfactant micelles. Langmuir 15, 6745-6754 (1999).

20. Hoang, T. K. N., Deriemaeker, L. \& Finsy, R. Ostwald ripening and solubilization in alkane in water emulsions stabilized by different surfactants. Phys. Chem. Chem. Phys. 6, 1413-1422 (2004).

21. Soto, R. \& Golestanian, R. Self-assembly of catalytically active colloidal molecules: tailoring activity through surface chemistry. Phys. Rev. Lett. 112, 068301 (2014).

22. Yalkowsky, S. H., He, Y. \& Jain, P. Handbook of Aqueous Solubility Data (CRC Press, 2016).

23. Carroll, B. J. The kinetics of solubilization of nonpolar oils by nonionic surfactant solutions. J. Colloid Interface Sci. 79, 126-135 (1981).

24. Donegan, A. C. \& Ward, A. J. Solubilization kinetics of $n$-alkanes by a non-ionic surfactant. J. Pharm. Pharmacol. 39, 45-47 (1987).

25. Wang, W., Chiang, T.-Y., Velegol, D. \& Mallouk, T. E. Understanding the efficiency of autonomous nano- and microscale motors. J. Am. Chem. Soc. 135, 10557-10565 (2013).

26. Bibette, J., Roux, D. \& Nallet, F. Depletion interactions and fluid-solid equilibrium in emulsions. Phys. Rev. Lett. 65, 2470 (1990).

27. Israelachvili, J. N. Intermolecular and Surface Forces (Academic, 2011).
28. Michelin, S., Lauga, E. \& Bartolo, D. Spontaneous autophoretic motion of isotropic particles. Phys. Fluids 25, 061701 (2013).

29. Palacci, J., Sacanna, S., Steinberg, A. P., Pine, D. J. \& Chaikin, P. M. Living crystals of light-activated colloidal surfers. Science 339, 936-940 (2013).

30. Rosslee, C. \& Abbott, N. L. Active control of interfacial properties. Curr. Opin. Colloid Interface Sci. 5, 81-87 (2000).

31. Thutupalli, S., Geyer, D., Singh, R., Adhikari, R. \& Stone, H. A. Flow-induced phase separation of active particles is controlled by boundary conditions. Proc. Natl Acad. Sci. USA 115, 5403-5408 (2018).

32. Illien, P., Golestanian, R. \& Sen, A. 'Fuelled' motion: phoretic motility and collective behaviour of active colloids. Chem. Soc. Rev. 69, 5508-5518 (2017).

33. Ross, T. D. et al. Controlling organization and forces in active matter through optically defined boundaries. Nature 572, 224-229 (2019).

34. Joseph, A. et al. Chemotactic synthetic vesicles: design and applications in blood-brain barrier crossing. Sci. Adv. 3, e1700362 (2017).

35. Somasundar, A. et al. Positive and negative chemotaxis of enzyme-coated liposome motors. Nat. Nanotechnol. 14, 1129-1134 (2019).

Publisher's note Springer Nature remains neutral with regard to jurisdictional claims in published maps and institutional affiliations.

(c) The Author(s), under exclusive licence to Springer Nature Limited 2020 


\section{Methods}

Chemicals. All the chemicals were used as received: Capstone FS-30, 1-(ethoxy) nonafluorobutanes (mixture of $n$ - and isobutyl isomers) and perfluorohexane(s) (98\%) (Synquest Laboratories), methoxyperfluorobutane (mixture of $n$ - and isobutyl isomers) (99\%), Triton X-100, 1-iodoheptane (copper stabilized, 98\%), 1-iodononane (copper stabilized, 98\%) and $n$-hexane (>99\%) (Sigma Aldrich), BOct (98\%), 1-iodobutane (copper stabilized, 99\%), 1-iodopentane (copper stabilized, 98\%), 1-iodohexane (copper stabilized, 98\%), 1-iodooctane (copper stabilized, 98\%), 1-iododecane (copper stabilized, 98\%), 1-iodododecane (copper stabilized, 98\%), 1-iodohexadecane (copper stabilized, 98\%) and n-octane (98\%) (Alfa Aesar), hexadecane (>98\%) (TCI), Lumogen F Red 305 (BASF), pyrromethene 650 (Exciton), polybead carboxylate microspheres $(0.97 \mu \mathrm{m}$ diameter, latex) (Polysciences, Inc.) and hydrophobic iron oxide nanoparticles (CMS Magnetics ferrofluid, particle-size unspecified, purchased on Amazon).

Droplet fabrication. The fabrication of monodisperse emulsion droplets was carried out using a four-channel flow focusing glass hydrophilic microfluidic chip with a channel depth of $100 \mu \mathrm{m}$ (Dolomite). Each microchannel inlet was connected to a reservoir of the desired liquid. The inlets for the inner fluid phases (for example, BOct or EFB) were connected to the reservoirs with polyether ether ketone tubing of inner diameter 0.0025 inch $(63.5 \mu \mathrm{m})$, outer diameter $1 / 16$ inch $(1.59 \mathrm{~mm})$ and in length 20 inches $(50.8 \mathrm{~cm})$, and the outer fluid phase that contained aqueous surfactant solution (for example, $0.5 \mathrm{wt} \%$ Triton) was connected to the reservoirs with polyether ether ketone tubing of inner diameter 0.005 inch $(127 \mu \mathrm{m})$, outer diameter $1 / 16 \mathrm{inch}(1.59 \mathrm{~mm})$ and length 20 inches $(50.8 \mathrm{~cm})$. The flow rates of each liquid were manipulated with a Fluigent MFSC-EZ pressure controller to the control droplet size. Typical pressures used for the inner phase fluids ranged from 1.0 to 3.0 bar and typical pressures for the outer phase fluids ranged from 0.50 to $6.0 \mathrm{bar}$.

\section{Droplet mixing protocol to observe two-body and multibody droplet} interactions. To observe and analyse droplet chasing interactions, a glass-bottomed dish of $1.5 \mathrm{inch}(3.8 \mathrm{~cm})$ diameter with aluminium walls was filled with approximately $2 \mathrm{ml}$ of surfactant solution and then approximately 100 prefabricated oil droplets of each type were transferred from a storage vial via a micropipette. The number of droplets was chosen to produce a dilute layer of droplets along the dish bottom (area fraction $<0.001$ ) necessary to analyse isolated chasing interactions between pairs of droplets. (The exception is the experiment shown in Supplementary Video 6, in which we examined the interactions among a high density of droplets.) The solution was then gently agitated for several seconds to randomly disperse the oil droplets throughout the dish. After dispersing the droplets, the dish was covered with a lid and left undisturbed for the remainder of the experiment. Droplets sedimented to the dish bottom within several seconds. Chasing interactions began within 1 minute and continued to grow in number over the course of the experiment. The formation of larger multidroplet clusters occurred more gradually over a period of minutes but continued to grow in size as long as the droplets remained active. Droplet motion and chasing interactions persisted as long as the predator droplets were not completely solubilized (typically on the order of an hour). By choosing oils that solubilized more slowly, or by starting with larger droplets, the motion could be extended for at least several hours before the predator droplets were solubilized completely. Droplets stored in saturated surfactant solutions for more than a week still exhibited chasing interactions when added to fresh surfactant solutions. Chasing interactions also still occurred even if the surfactant solution was pre-saturated with the two oils.

Droplet imaging. Droplet interactions were observed using an inverted optical microscope (Nikon, Eclipse Ti-U) in brightfield transmission mode between $\times 2$ and $\times 40$ magnification. Images were recorded using an attached camera (Andor, Zyla sCMOS) in video mode with a range from 0.1 to 25 frames per second. For fluorescence imaging, a TRITC filter set was used (Nikon, centre wavelength for excitation $540 \mathrm{~nm}$, and for emission, $605 \mathrm{~nm}$ ) along with a Nikon Plan Fluor $\times 20$ objective. To distinguish droplets of similar refractive index and verify the chasing direction, Lumogen F Red 305 fluorescent dye was added to one droplet type and an overlay of brightfield and fluorescence imaging was used (see, for example, Extended Data Fig. 3). Controls were conducted to verify that the dye did not affect the chasing dynamics.

Observation of oil exchange. To observe the exchange of oil between BOct and EFB droplets, as shown in Fig. 1d, a single depression microscope slide (AmScope) was completely submerged in a large dish that that contained a $0.5 \mathrm{wt} \%$ Triton surfactant solution. An approximately equal number of BOct and EFB droplets of about $70 \mu \mathrm{m}$ diameter were added via a micropipette to the centre of the slide depression, where they were spatially confined in a circular monolayer of $1 \mathrm{~cm}$ diameter. Droplets were then monitored for a period of more than 4 hours. Over time, the EFB droplets (refractive index, $n=1.282$ ) visually disappeared due to index matching with the surrounding aqueous phase $(n \approx 1.33)$ caused by the uptake of the higher index BOct oil $(n=1.453)$. Over a similar time period, BOct droplets across the cluster were found to shrink noticeably as their oil was more readily solubilized by the Triton surfactant micelles. Exchange between BOct and EFB droplets in $5.0 \mathrm{wt} \%$ Capstone was conducted in the same experimental set-up as described above. As shown in Extended Data Fig. 1, BOct droplets visually disappeared due to index matching with the aqueous phase caused by an uptake of the lower index EFB oil, which was preferentially solubilized by the fluorinated Capstone surfactant.

Control experiments were conducted to rule out the possibility that changes in droplet refractive index resulted from surfactant partitioning or from inverse micelles; EFB and BOct were equilibrated with $0.5 \mathrm{wt} \%$ Triton and the refractive index differed from the pure oils by $\Delta n<0.001$.

The transfer of a fluorescent dye between droplets was also used to support the role of surfactant-mediated oil exchange. 1-iododecane droplets prepared with a $0.1 \mathrm{wt} \%$ pyrromethene 650 fluorescent dye were added to a dish that contained 1-iodohexane droplets without dye. As shown in Extended Data Fig. 4 , the 1-iodohexane droplets increased in fluorescence over time. Time series fluorescent images were obtained every 5-10 min. over the course of 1 hour with the fluorescent lamp turned off between measurements to reduce possible photobleaching.

Measurement of refractive indices and oil miscibility. The miscibilities of fluorinated oils and BOct were analysed based on refractive index measurements performed using a temperature-controlled J457FC refractometer (Rudolph Research) at $20^{\circ} \mathrm{C}$. BOct (refractive index $n=1.453$, measured; $n=1.450-1.463$ in the literature ${ }^{36}$, at $\left.20^{\circ} \mathrm{C}\right)$ and $\operatorname{EFB}(n=1.282$, measured) were completely miscible at room temperature, and the refractive index of their binary mixtures varied linearly as a function of the volume fraction of each oil. Given this observation, we presumed that a similar linear trend would hold for binary mixtures of other hydrocarbon and fluorocarbon oils and that refractive index could be used to quantify the degree of miscibility by extrapolating between the indices of the two pure oils. Methoxyperfluorobutane ( $n=1.271$, measured) was saturated with BOct and the refractive index of the mixture was measured $(n=1.302)$ and yielded an estimated miscibility of $\frac{1.302-1.271}{1.453-1.271}=0.17$, or $17 \%$. Perfluorohexane $(n=1.254$, measured; $n=1.252$ in the literature ${ }^{36}$ at $22^{\circ} \mathrm{C}$ ) was saturated with BOct and the refractive index of the mixture was measured $(n=1.255)$ and yielded an estimated miscibility of $\frac{1.255-1.254}{1.453-1.254}=0.005$, or $0.5 \%$.

Observation and characterization of flow profiles. The flows inside chasing iodoheptane and iodohexane droplets were visualized by dispersing a $0.5 \mathrm{vol} \%$ hydrophobic iron oxide nanoparticle solution ('Chemicals' above) in the oil phase prior to emulsification. This combination of oils was chosen because it was found to readily disperse the hydrophobic iron oxide nanoparticles. The particles were dispersed in each oil prior to the emulsification of droplets using microfluidics. After emulsification, the droplets were mixed in a $0.5 \mathrm{wt} \%$ Triton surfactant solution following the protocol described in 'Droplet mixing protocol for observing two-body and multibody droplet interactions' (above). The iodoheptane droplets were found to chase the iodohexane droplets, similar to the experiments without iron oxide particles (Extended Data Fig. 3), which suggests that the iron oxide particles did not have a substantial effect on the droplet interfaces and were inert tracers of flow. When the two types of droplets chased each other, asymmetric flow profiles were observed in both droplets (Supplementary Video 5), consistent with the flow illustrated in Fig. 2e.

The flows outside the chasing iodoheptane and iodohexane droplets were visualized by using latex microspheres as tracer particles suspended in a $0.5 \mathrm{wt} \%$ Triton surfactant solution (Supplementary Video 5). Droplets emulsified in the absence of tracer particles were added to the $0.5 \mathrm{wt} \%$ Triton surfactant solution following the protocol described in 'Droplet mixing protocol for observing two-body and multibody droplet interactions' (above). The particle movement that surrounded the droplets was recorded at a rate of 25 frames per second. Flow velocities were measured using the Matlab 2019b particle image velocimetry implementation PIVlab version $2.31^{37}$. All the frames were rotated so that the line that connects the centres of the predator and prey droplets was aligned with the $x$ axis and subsequently averaged to obtain the flow profile shown in Extended Data Fig. 5.

Oil solubilization rate measurement. Oil solubilization rates were determined by monitoring the sizes of individual isolated droplets (starting diameter, $60-80 \mu \mathrm{m}$ ) in different surfactant solutions over a period of at least $20 \mathrm{~min}$. Time-lapse images were analysed with calibrated pixel values using the Nikon Elements-D software suite. The rate of droplet diameter change was found to be constant over the observation period, which indicates an interfacially limited solubilization process, consistent with previous kinetic solubilization studies on non-ionic surfactants ${ }^{23}$. Solubilizing droplets smaller than $40 \mu \mathrm{m}$ often became self-propelled, as characterized by their persistent directional motion even in the absence of other surrounding droplets (Extended Data Fig. 6). Solubilizing droplets were previously studied and exhibited self-propelled motion spontaneously above a critical Péclet number ${ }^{11,28}$. Owing to their advection, self-propelled droplets were found to solubilize faster than stationary droplets and were therefore excluded from the measurements of solubilization rate. 
Interfacial tension measurement. Oil-water interfacial tensions were obtained by the pendant drop method using a Ramé Hart 250 Automatic Goniometer. A 28-gauge stainless-steel probe tip needle was used to dispense oil drops into a glass cuvette filled with approximately $5 \mathrm{ml}$ of aqueous surfactant solution. The DROPimage Advanced analysis software package was used to analyse images of drop shape to determine the interfacial tension values based on measuring the curvature deformation of the drop in response to gravitational and buoyant forces. The interfacial tension of BOct and EFB was measured in a $0.5 \mathrm{wt} \%$ Triton solution, as well as in a BOct-saturated $0.5 \mathrm{wt} \%$ Triton solution. The BOct-saturated surfactant solution was prepared by emulsifying an excess volume of BOct oil ( $>5 \mathrm{vol} \%$ ) in $0.5 \mathrm{wt} \%$ Triton and allowing it to equilibrate for more than 72 hours before measurement. The BOct oil for all the experiments was itself was also pre-equilibrated with $0.5 \mathrm{wt} \%$ Triton for one week prior to interfacial tension measurements to account for the possible surfactant partitioning into the oil ${ }^{38}$. A single dynamic interfacial tension measurement curve is shown for each condition in Extended Data Fig. 2. At least three measurements were conducted for each condition, and elevated interfacial tension values for the BOct-saturated solutions were observed in all cases.

Image analysis and droplet tracking. To quantify the dynamics of chasing droplets, we identified droplet positions using the Matlab imfindcircle algorithm, which is based on a Hough transformation. Subsequent tracking of the positions was performed using the 2007 Matlab implementation by Blair and Dufresne of the Crocker and Grier tracking software ${ }^{39}$, which was downloaded from http://site. physics.georgetown.edu/matlab/. Matlab version R2018B was used.

\section{Data availability}

All relevant data generated or analysed for this study are included in the published article and its Supplementary Information files.

\section{References}

36. Wohlfahrt, C. \& Wohlfahrt, B. Refractive Indices of Organic Liquids: Optical Constants (Springer, 1996).

37. Thielicke, W. \& Stamhuis, E. J. PIVlab-time-resolved digital particle image velocimetry tool for MATLAB (programmed in MATLAB 7, R14) (BSD license, 2014).
38. Ravera, F., Ferrari, M., Liggieri, L., Miller, R. \& Passerone, A. Measurement of the partition coefficient of surfactants in water/oil systems. Langmuir 13, 4817-4820 (1997).

39. Crocker, J. C. \& Grier, D. G. Methods of digital video microscopy for colloidal studies. J. Colloid Interface Sci. 179, 298-310 (1996).

\section{Acknowledgements}

We thank C. Wentworth for her help obtaining interfacial tension measurements using the pendant drop method. L.D.Z., C.H.M. and Y.-J.C. acknowledge support from the Army Research Office through grant no. W911NF-18-1-0414 and the Penn State MRSEC funded by the National Science Foundation (DMR-1420620). C.H.M. acknowledges support from the Thomas and June Beaver Fellowship at Penn State and the Pennsylvania Space Grant Fellowship, and Y.-J.C. received support from the Erickson Discovery Grant Program at Penn State. P.G.M. acknowledges funding from the NWO (Dutch National Science Foundation) Graduate Program through the Debye Institute for Nanomaterials. J.G. wishes to thank the program for Chang Jiang Scholars and Innovative Research Teams in Universities (no. IRT 17R40) and the 111 Project of the PRC.

\section{Author contributions}

C.H.M., P.G.M. and L.D.Z. developed the concept for the research. C.H.M and Y.-J.C. conducted the droplet chasing experiments under different conditions and measured the oil solubilization rates. P.G.M. analysed data on the droplet motions and performed the simulations. C.H.M., P.G.M. and L.D.Z. wrote the manuscript. P.G.M. was supervised by W.K.K., J.G. and A.v.B. C.H.M. and Y-J.C. were supervised by L.D.Z. All the authors discussed the results and manuscript.

\section{Competing interests}

The authors declare no competing interests.

\section{Additional information}

Extended data is available for this paper at https://doi.org/10.1038/s41557-020-00575-0.

Supplementary information is available for this paper at https://doi.org/10.1038/ s41557-020-00575-0.

Correspondence and requests for materials should be addressed to L.D.Z.

Reprints and permissions information is available at www.nature.com/reprints. 


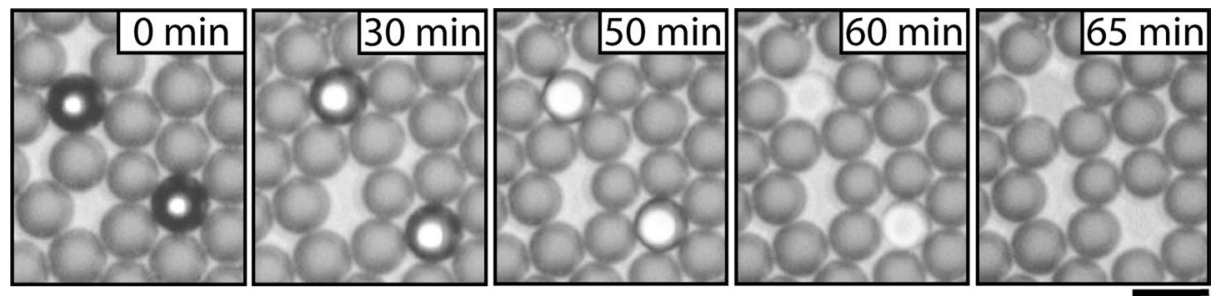

Extended Data Fig. 1 | Observation of fluorinated oil transfer between droplets in Capstone surfactant solution. The fluorosurfactant Capstone FS-30 preferentially solubilizes EFB over BOct (rate of $0.54 \mu \mathrm{m} / \mathrm{min}$ vs. $0.01 \mu \mathrm{m} / \mathrm{min}$, respectively, in $5.0 \mathrm{wt} \%$ Capstone) and facilitates the net directional transfer of EFB oil into BOct droplets. BOct $(n=1.45)$ droplets surrounded by EFB in 5.0 wt\% Capstone undergo a shift their appearance as the droplets' refractive index changes due to the addition of $\operatorname{EFB}(n=1.28)$, eventually becoming nearly transparent due to index matching with the aqueous phase $(n=1.33)$. Scale, $100 \mu \mathrm{m}$. 
a

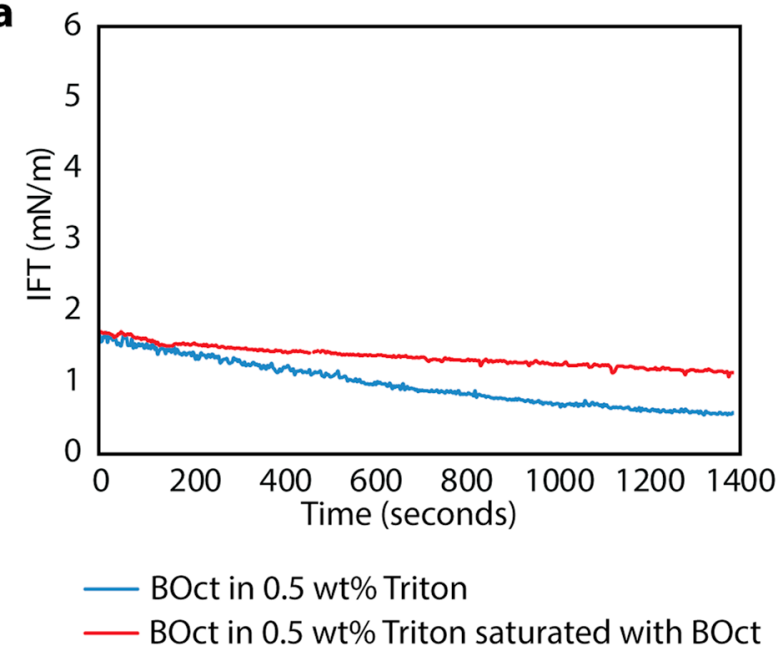

b

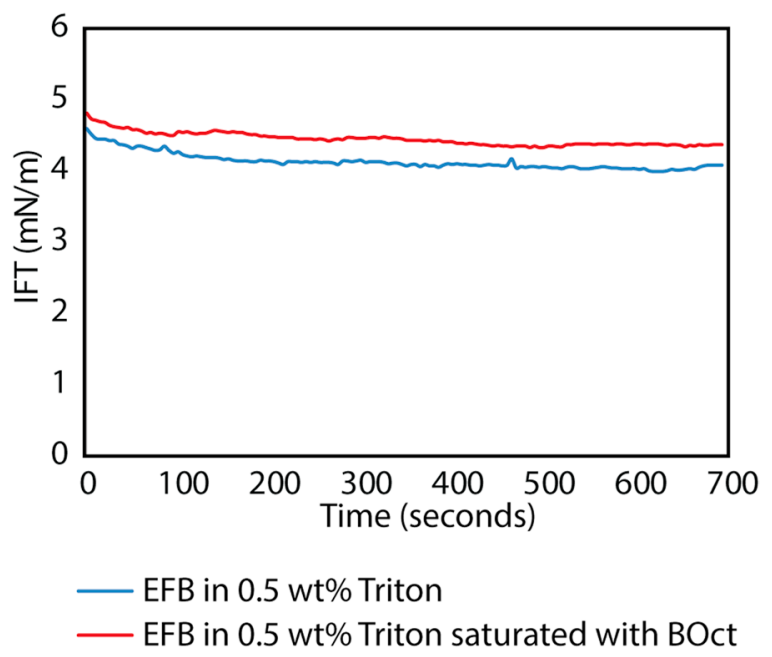

Extended Data Fig. 2 | The pendant drop method is used to measure the dynamic interfacial tension (IFT) increase resultant from BOct oil saturation in Triton surfactant solutions. a, The interfacial tension of BOct is higher in BOct-saturated $0.5 \mathrm{wt} \%$ Triton surfactant solution (red) compared to unsaturated surfactant solution (blue). b, A higher interfacial tension was also observed for EFB in BOct-saturated 0.5 wt $\%$ Triton (red), compared to oil-free Triton (blue). Experiments were produced in triplicate and the same trends were consistently observed. 

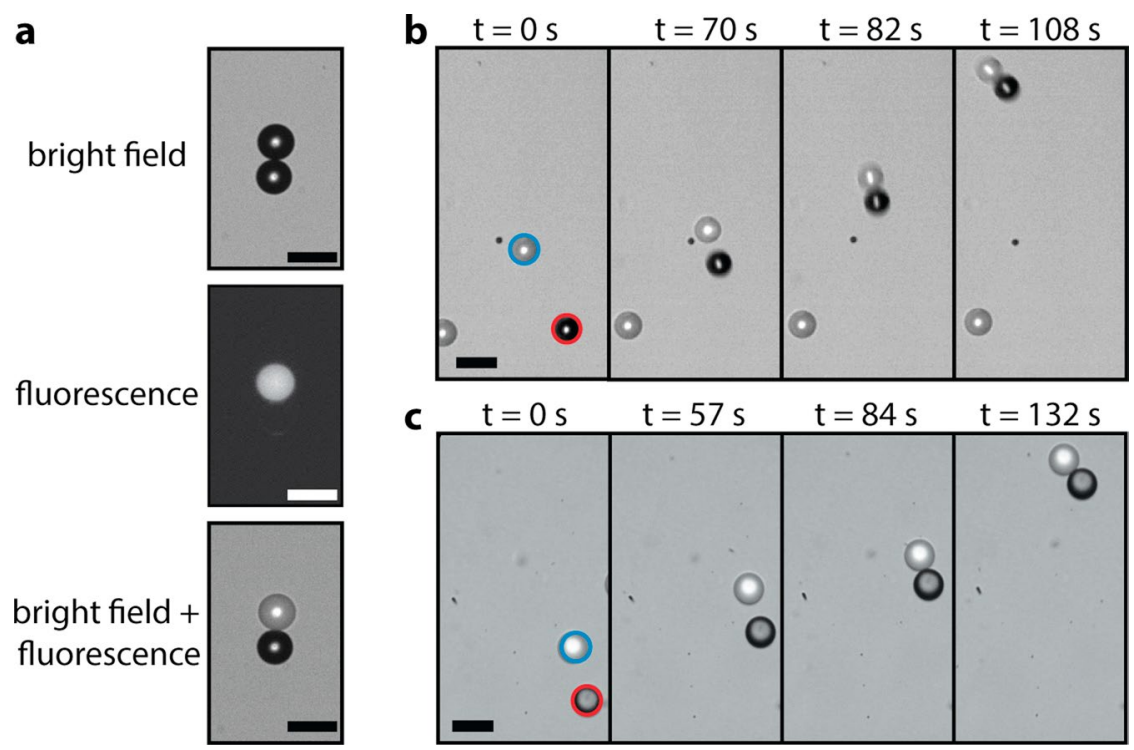

Extended Data Fig. 3 | Fluorescence was used to distinguish between chasing iodoalkanes or chasing alkanes and identify predator and prey. a, Under transmission brightfield imaging, iodohexane and iodoheptane droplets are indistinguishable (top, and Supplementary Video 4); by dying the iodohexane droplet with fluorescent Lumogen F Red 305, the iodohexane droplet can be easily identified using fluorescence microscopy (middle). By simultaneously imaging the droplets in both transmission brightfield and fluorescence (bottom), we can visualize all droplets and also distinguish the two types of oil. This imaging method is used in (b,c). b. An iodoheptane droplet (outlined in red) chases an iodohexane droplet dyed with fluorescent Lumogen F Red 305 (outlined in blue) in $0.5 \mathrm{wt} \%$ Triton. The optical micrographs were taken with simultaneous transmission brightfield and fluorescence imaging as shown in (a). Scale, $100 \mu \mathrm{m}$. c, An octane droplet (outlined in red) chases a hexadecane droplet dyed with fluorescent Lumogen F Red 305 (outlined in blue) in 0.5 wt $\%$ Triton. Optical micrographs were taken with simultaneous transmission brightfield and fluorescence imaging as shown in (a). Scale, $100 \mu \mathrm{m}$. 

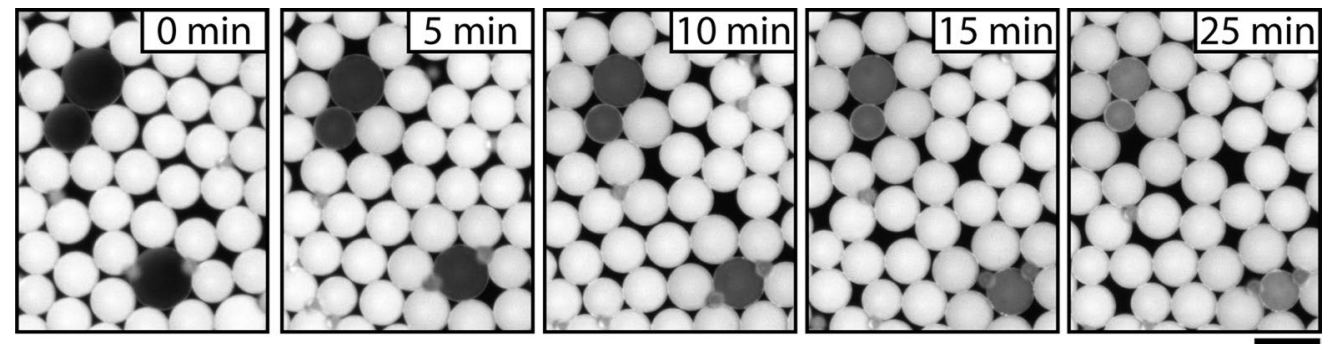

Extended Data Fig. 4 | Fluorescence was used to observe the exchange of oil between iodoalkane droplets. 1-iododecane droplets prepared with fluorescent pyrromethene 650 dye were mixed with 1-iodohexane droplets in $0.5 \mathrm{wt} \%$ Triton. 1-iodohexane droplets became increasingly fluorescent over time due to the surfactant-mediated transfer of dye, which we use to infer that transfer of oil also occurs. The 1-iodohexane droplets shrink faster than 1-iododecane droplets due to faster solubilization into the aqueous phase $(0.41 \mu \mathrm{m} / \mathrm{min}$ vs. $0.24 \mu \mathrm{m} / \mathrm{min})$. Scale, $100 \mu \mathrm{m}$. 


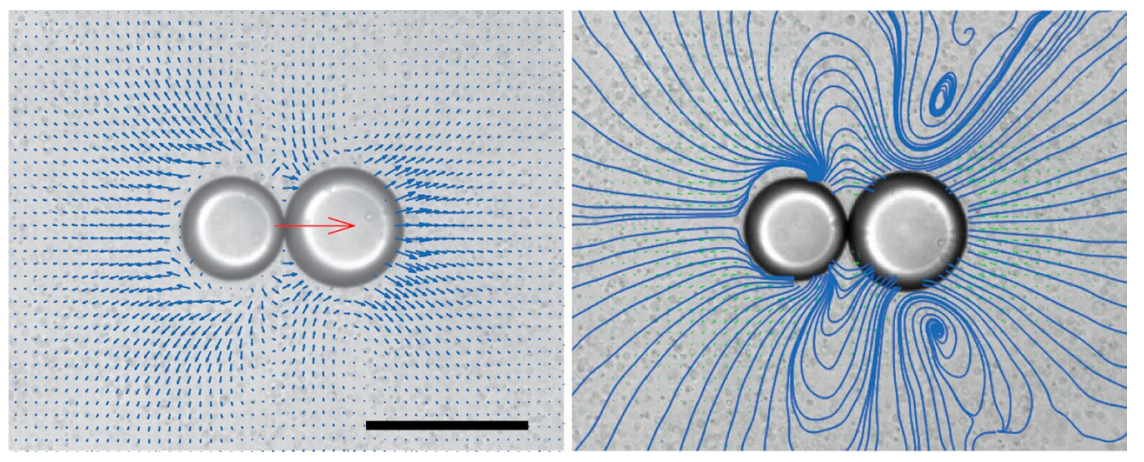

Extended Data Fig. 5 | External flow profile surrounding chasing iodoalkane droplet pair. External flow field surrounding a chasing pair of 1-iodoheptane and 1-iodohexane droplets in $0.5 \mathrm{wt} \%$ Triton X-100 was visualized using neutrally buoyant tracer microparticles. The direction of pair motion is from left to right and 1-iodoheptane is the predator. The blue arrows in the left panel indicate the average local flow velocity of the fluid. Before averaging, each frame was rotated so that the line connecting the predator and prey droplets aligned with the x-axis. The red arrow indicates the average velocity of the chaser, as seen in Supplementary Video 5, v =11.1 $\frac{\mathrm{\mu m}}{\mathrm{s}}$ The length of the blue arrows is scaled to the velocity of the droplet pair. The panel on the right shows streamlines around the same droplet pair. Scale bar is $100 \mu \mathrm{m}$. 


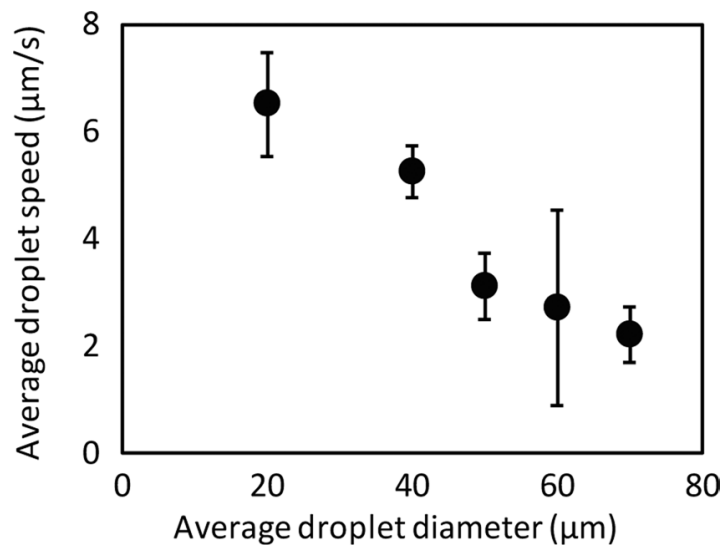

Extended Data Fig. 6 | BOct droplets become self-propelled at smaller diameters. Droplet-tracking image analysis was used to determine the average droplet speed of BOct droplets as a function of droplet diameter as the drops were solubilizing in $0.5 \mathrm{wt} \%$ Triton. The average speed was calculated by measuring the nominal speed and subtracting the component of the speed that all droplets had in the same direction due to drift. Each data point represents between 20 and 50 droplet measurements, and the error bars represent the standard deviation. As the droplets become smaller, the average self-propulsion speed of the BOct droplets increases. 


\section{NATURE CHEMISTRY}
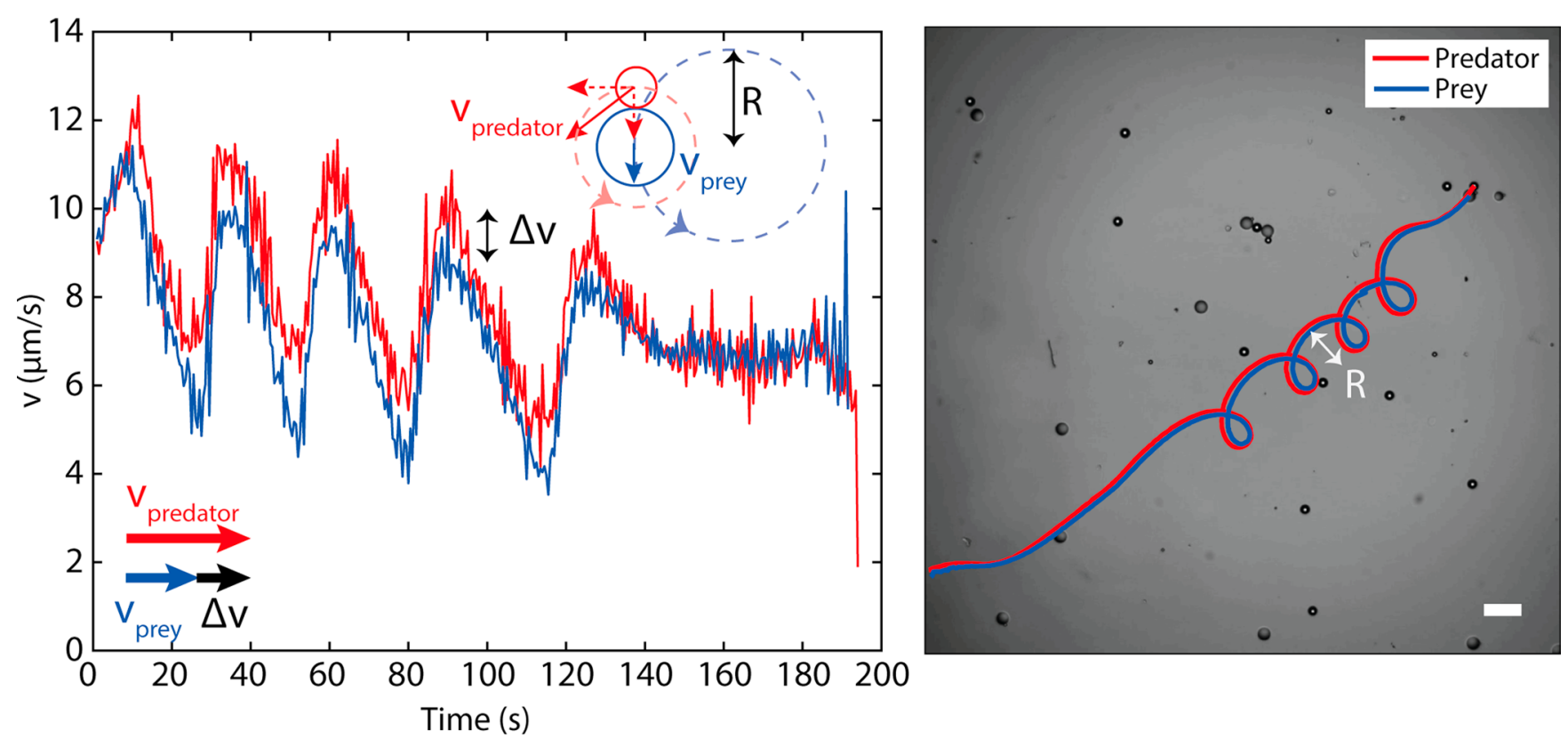

Extended Data Fig. 7 | Trochoidal motion of a swimming predator-prey pair. a, Speed of the predator BOct droplet (red) and prey methoxyperfluorobutane droplet (blue) as function of time. The schematic indicates how self-propulsion of the predator drop along the surface of the prey results in a circular trajectory of the chasing pair. b, Trochoidal trajectory of a BOct droplet chasing after an methoxyperfluorobutane droplet. Scale, $100 \mu \mathrm{m}$. 